\title{
Lead Site CV6LU
}

National Cancer Institute

\section{Source}

National Cancer Institute. Lead Site CV6LU. NCI Thesaurus. Code C90407.

V2 electrode (+) placed in the 6th intercostal space on the left side of the thorax along a line parallel to the level of the point of the shoulder. 